\title{
Case Report \\ Polyserositis: An Extremely Rare Life-Threatening Manifestation of Pheochromocytoma
}

\author{
Maryam Heidarpour $\mathbb{D D}^{1}$ Mohammad Ali Haghighatpanah ${ }^{1}{ }^{2}{ }^{2}$ Hassan Rezvanian (D), \\ Motahare Yadegarfar $\mathbb{D}^{\mathrm{D}}{ }^{3}$ Amir Mohammad Mozafari, ${ }^{4}$ Mehrbod Vakhshoori $\mathbb{D}^{5}{ }^{5}$ \\ and Davood Shafie ${ }^{5}{ }^{5}$ \\ ${ }^{1}$ Isfahan Endocrine and Metabolism Research Center, Isfahan University of Medical Sciences, Isfahan, Iran \\ ${ }^{2}$ Isfahan University of Medical Sciences, Isfahan, Iran \\ ${ }^{3}$ University Hospitals of Leicester NHS Foundation Trust, LE1 5WW Leicester, UK \\ ${ }^{4}$ Medical Library and Information Sciences Department, Health Information Technology Research Center, \\ School of Management and Medical Information Sciences, Isfahan University of Medical Sciences, Isfahan, Iran \\ ${ }^{5}$ Heart Failure Research Center, Cardiovascular Research Institute, Isfahan University of Medical Sciences, Isfahan, Iran \\ Correspondence should be addressed to Davood Shafie; d.shafie87@gmail.com
}

Received 15 April 2020; Revised 25 May 2020; Accepted 5 June 2020; Published 6 July 2020

Academic Editor: Suat Simsek

Copyright (c) 2020 Maryam Heidarpour et al. This is an open access article distributed under the Creative Commons Attribution License, which permits unrestricted use, distribution, and reproduction in any medium, provided the original work is properly cited.

\begin{abstract}
The pericardium is an uncommon site for manifestation of pheochromocytoma. Herein, the case of a 57-year-old man with cardiac tamponade is presented. Pericardiocentesis was performed, and the vital signs were stabilized afterwards. An abdominal computed tomography (CT) scan illustrated a nonhomogeneous right adrenal mass suspicious of pheochromocytoma, planned for right adrenalectomy. He recovered well after surgery, and his subsequent follow-ups did not reveal any complications.
\end{abstract}

\section{Introduction}

Pheochromocytoma is a rare catecholamine-secreting tumor originating from adrenal chromaffin cells. The incidence of this tumor is approximately 0.8 per 100,000 people per year [1]. The classic triad of this tumor includes headache, palpitations, and sweating; however, it can present with a wide range of clinical symptoms; therefore, it is also referred as a great masquerader [2]. Pheochromocytoma increases morbidity and mortality by causing cardiovascular complications, but due to the rareness of this tumor, most cardiovascular complications related to the aforementioned disease are limited to case reports or series [3]. In this study, we presented a patient who initially referred with severe shortness of breath, caused by cardiac tamponade, in which further investigations revealed the presence of one of the rarest complication of pheochromocytoma.

\section{Case Presentation}

A 57-year-old man with a remote history of hypertension (HTN) presented to the emergency department with the chief complaint of shortness of breath. His medical history did not reveal any positive findings attributed to the probable occurrence of coronary artery diseases. He had no previous history of a sudden increase in blood pressure, palpitations, sweating, and headache. Initial vital signs included blood pressure $90 / 70 \mathrm{mmHg}$, a heart rate of 109 beats per minute $(\mathrm{bpm})$, respiratory rate of 19 per minute, $\mathrm{O}_{2}$ saturation of $97 \%$ on room air, and temperature of $37.1^{\circ} \mathrm{C}$. On examination, he was found to have distended neck veins, tachycardia, and muffled heart sounds. The result of electrocardiogram (ECG) was not indicative of ischemic heart diseases. Echocardiography showed a normal left ventricular ejection fraction (LVEF) and massive pericardial effusion with diastolic right ventricular collapse without any regional 


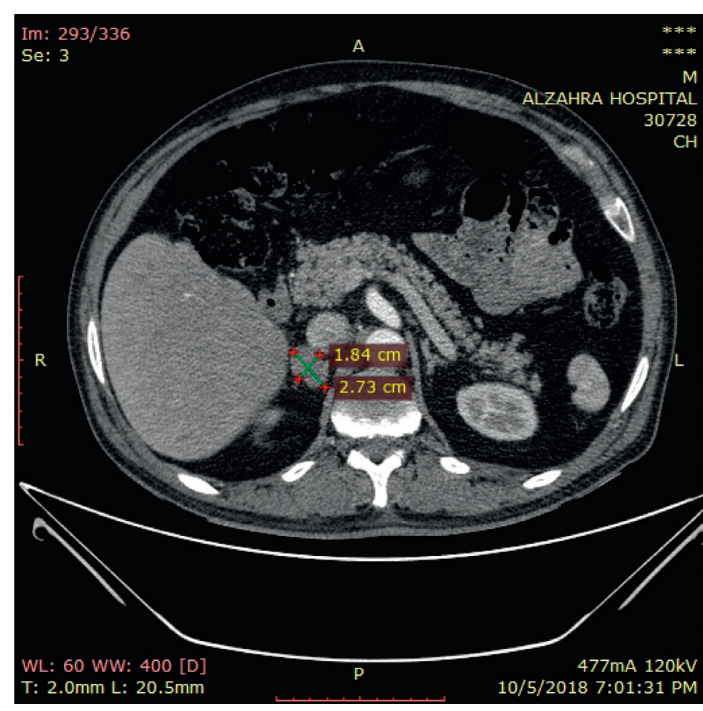

(a)

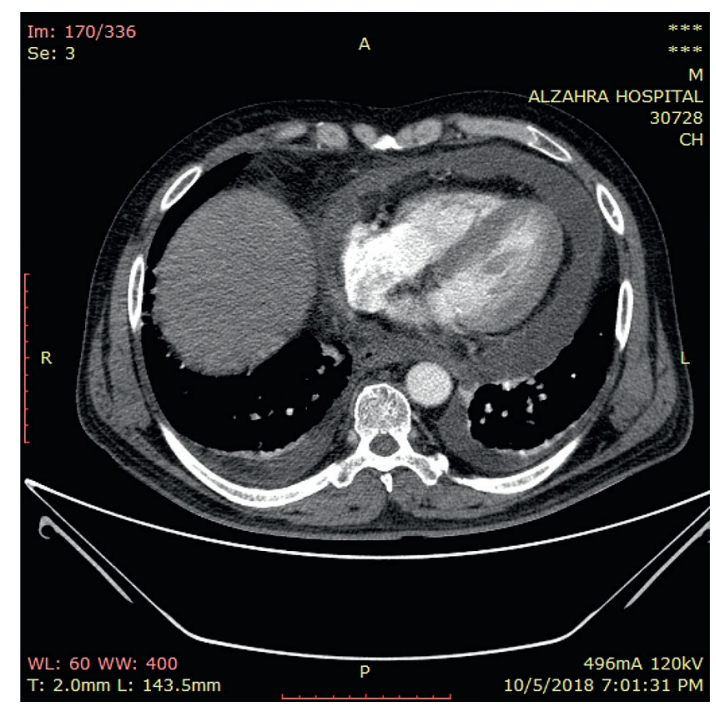

(b)

Figure 1: Chest and abdominal CT scan of the patient shows heterogeneous right adrenal mass (a) and pericardial and bilateral pleural effusion (b).

wall motion abnormalities as well as positive findings in regards to takotsubo cardiomyopathy. Chest X-ray demonstrated large bilateral pleural effusion. He was scheduled for urgent pericardiocentesis by the subxiphoid approach, and 600 milliliters of serous pericardial fluid was drained; his symptoms were relieved. Chest tubes were also inserted, and approximately 750 milliliters of nonbloody fluid was drained. As of suspicion to pericarditis, colchicine and ibuprofen were prescribed for the patient. On the fifth day of admission, the pericardial effusion reaccumulated in spite of prolonged pericardial drainage. Therefore, a subxiphoid pericardial window with pericardial biopsy was performed. Pericardial cytology was nonhemorrhagic, and the microbiology test was negative. The histological study showed reactive mesothelial hyperplasia.

On day 7, chest and abdominal computed tomography (CT) scan was performed for further evaluation, which showed a heterogeneous right adrenal mass measuring $27 * 18 \mathrm{~mm}$. The adrenal mass on the unenhanced images had a density of 70 Hounsfield units (Figure 1). Given these findings, pheochromocytoma and adrenocortical carcinoma were considered to be the most probable differential diagnoses for this adrenal mass. Laboratory values were notable for a 24-hour urine metanephrine of $260 \mu \mathrm{g} /$ day (normal $<315 \mu \mathrm{g} /$ day), normetanephrine of $510 \mu \mathrm{g} /$ day (normal $<670 \mu \mathrm{g} /$ day), adrenaline of $6.89 \mu \mathrm{g} /$ day (normal $<20 \mu \mathrm{g} /$ day), noradrenaline of $171 \mu \mathrm{g} /$ day (normal $<90 \mu \mathrm{g} /$ day), free cortisol of $23 \mu \mathrm{g} /$ day (normal: $3.5-45 \mu \mathrm{g} /$ day), serum aldosterone of $8 \mathrm{ng} / \mathrm{dl}$ (normal: $2-9 \mathrm{ng} / \mathrm{dl}$ ), plasma renin activity of $1.15 \mathrm{ng} / \mathrm{ml} / \mathrm{h}$ (normal: $1.1-1.28 \mathrm{ng} / \mathrm{ml} / \mathrm{h}$ ), and $17-\mathrm{OH}$ progesterone of $125 \mathrm{ng} / \mathrm{dl}$ (normal: less than $200 \mathrm{ng} / \mathrm{dL}$ ). All other hematological and biochemical tests were in normal ranges. These findings were consistent with the diagnosis of pheochromocytoma. He was started on phenoxybenzamine $10 \mathrm{mg}$ twice a day, and the dosage was increased by $10 \mathrm{mg}$ every three days to a maximum dosage of $20 \mathrm{mg}$ twice a day. One week afterwards, propranolol with a dose of $10 \mathrm{mg}$ every 8 hours was prescribed. Then, right adrenalectomy was performed without any complications, and the postoperative histopathologic report was in agreement with a diagnosis of pheochromocytoma (Figure 2). No complications were reported in the postoperative period. He was followed in an outpatient setting on a regular basis. Three months after adrenalectomy, the patient had no evidence of the disease. The latest echocardiography showed the LVEF to be $55 \%$. Other echocardiographic measurements were within the normal values.

\section{Discussion}

The presented case was a middle-aged patient with a history of HTN who presented with pleural and pericardial effusions and highlights the point that the clinical presentation of pheochromocytoma could be varied significantly. Therefore, clinical suspicion of this disease is the key to a timely diagnosis [2]. Pheochromocytoma is a neoplasm of adrenal chromaffin cells producing excess catecholamines. It is estimated that at least $25 \%$ of the patients has never being diagnosed during their lifetimes [4]. Furthermore, pheochromocytoma has various cardiovascular manifestations, in which hypertension is categorized as the most common one. Other possible cardiovascular presentations include myocardial infarction, cardiac hypertrophy, systolic and diastolic heart failure, arrhythmia, myocarditis, and pericardial effusion [1]. The pericardium is a rare site for primary tumors or the metastasis of malignant neoplasms. Few studies revealed that pericardial effusion is a very rare manifestation of pheochromocytoma [4-6]. It is postulated that the elevated levels of catecholamines increase the level of inflammatory factors leading to inflammatory symptoms such as fever, weight loss, and polyserositis [7-11]. Westfried et al. reported a case of malignant pheochromocytoma presented 


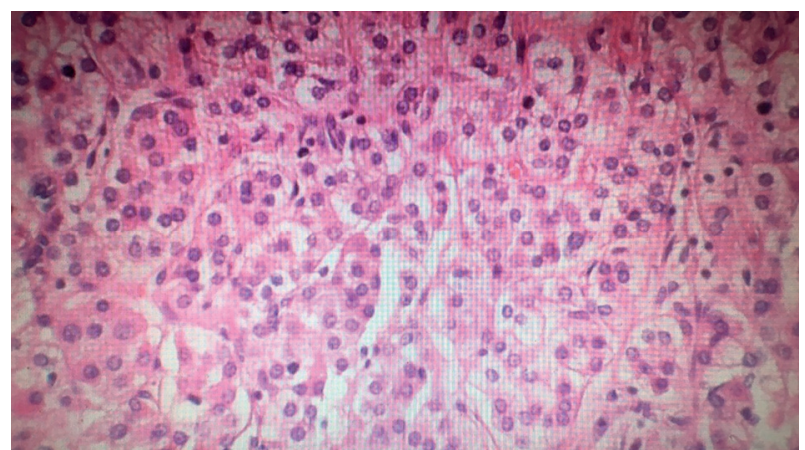

Figure 2: Histopathology of the adrenal mass. Polygonal cells with abundant granular amphophilic cytoplasm arranged in well-defined nests (zellbalen appearance).

with shortness of breath with demonstration of metastasis to pericardium in a histopathologic sample [6]. In our case, however, catecholamine-induced pericardial and pleural effusions were the first manifestation leading to further evaluation and prompt diagnosis of pheochromocytoma. As mentioned above, the histopathologic review showed only an inflammatory reaction. To our best knowledge, there is no published case report, in which pheochromocytoma would be presented with reactive pericardial and pleural effusion as the first manifestation, bearing in mind that pheochromocytoma can manifest with unusual presentations. Therefore, it is expected that only a small percentage of severe cases of pheochromocytoma would be diagnosed or reported [4]. Ferreira et al. designed a study in order to characterize cardiac involvement in sixty patients suffering from pheochromocytoma using cardiac magnetic resonance (CMR). They found that cardiac involvement, including impaired systolic and diastolic function, pericardial effusion, myocarditis, and markers for focal and diffuse fibrosis are common among these patients [1]. Indeed, some abnormalities such as subclinical systolic and dysfunction and markers for fibrosis might be persisted for a long time in spite of the curative surgery of the pheochromocytoma without any evidence of underlying disease. Thus, it seems that although pheochromocytoma is a rare disease, delayed diagnosis could be life-threatening [3].

In conclusion, it should be considered that clinical suspicion of pheochromocytoma is a key for a timely diagnosis, and more extensive studies are required to determine the actual rate of cardiovascular involvement in the acute phase of pheochromocytoma and after the curative surgery.

\section{Ethical Approval}

Ethical approval is not necessary for retrospective studies and case presentations in our institutional policies. However, informed consent had been obtained from the patient to publish this material.

\section{Conflicts of Interest}

The authors declare that they have no conflicts of interest.

\section{Acknowledgments}

This research did not receive any specific grant from any funding agency in the public, commercial, or not-for-profit sector.

\section{References}

[1] V. M. Ferreira, M. Marcelino, S. K. Piechnik et al., "Pheochromocytoma is characterized by catecholamine-mediated myocarditis, focal and diffuse myocardial fibrosis, and myocardial dysfunction," Journal of the American College of Cardiology, vol. 67, no. 20, pp. 2364-2374, 2016.

[2] H. A. Reyes, J. J. Paquin, and D. M. Harris, "Pheochromocytoma, "the great masquerader," presenting as severe acute decompensated heart failure in a young patient," Case Reports in Cardiology, vol. 2018, Article ID 8767801, 5 pages, 2018.

[3] L. R. Leite, P. G. Macedo, S. N. Santos, L. Quaglia, C. E. Mesas, and A. De Paola, "Life-threatening cardiac manifestations of pheochromocytoma," Case Reports in Medicine, vol. 2010, Article ID 976120, 4 pages, 2010.

[4] H. Falhammar, M. Kjellman, and J. Calissendorff, "Initial clinical presentation and spectrum of pheochromocytoma: a study of 94 cases from a single center," Endocrine Connections, vol. 7, no. 1, pp. 186-192, 2018.

[5] W. P. Skelton, D. Mahtta, S. Welniak, A. J. Franke, and L. H. Dang, "Pericardial effusion as an atypical initial presentation of extragonadal nonseminomatous germ cell tumor: a case report and literature review," Oxford Medical Case Reports, vol. 2018, no. 2, Article ID omx097, 2018.

[6] M. Westfried, D. Mandel, M. N. Alderete, J. Groopman, and S. Minkowitz, "Sipple's syndrome with a malignant pheochromocytoma presenting as a pericardial effusion," Cardiology, vol. 63, no. 5, pp. 305-311, 1978.

[7] S. Yarman, O. Soyluk, E. Altunoglu, and R. Tanakol, "Interleukin-6-producing pheochromocytoma presenting with fever of unknown origin," Clinics, vol. 66, no. 10, pp. 1843-1845, 2011.

[8] M. Ciacciarelli, D. Bellini, A. Laghi et al., "IL-6-producing, noncatecholamines secreting pheochromocytoma presenting as fever of unknown origin," Case Reports in Medicine, vol. 2016, Article ID 3489046, 5 pages, 2016.

[9] X. Cheng, M. Zhang, Y. Xiao, H. Li, Y. Zhang, and Z. Ji, "Interleukin-6-producing pheochromocytoma as a new reason for fever of unknown origin: a retrospective study," Endocrine Practice, vol. 24, no. 6, pp. 507-511, 2018.

[10] Y. Kawahara, A. Morimoto, A. Onoue, Y. Kashii, N. Fukushima, and Y. Gunji, "Persistent fever and weight loss due to an interleukin-6-producing adrenocortical oncocytoma in a girl: review of the literature," European Journal of Pediatrics, vol. 173, no. 8, pp. 1107-1110, 2014.

[11] T. Zelinka, O. Petrák, B. Štrauch et al., "Elevated inflammation markers in pheochromocytoma compared to other forms of hypertension," Neuroimmunomodulation, vol. 14, no. 1, pp. 57-64, 2007. 